\title{
O. Külpe, Grundriss des Psychologie
}

1893

\section{Alain Flajoliet}

\section{OpenEdition}

\section{Journals}

Édition électronique

URL : http://journals.openedition.org/alter/1808

DOI : $10.4000 /$ alter.1808

ISSN : 2558-7927

Éditeur :

Association ALTER, Archives Husserl (CNRS-UMR 8547)

\section{Édition imprimée}

Date de publication : 1 octobre 2010

Pagination : 355-365

ISBN : 2-9522374-6-8

ISSN : 1249-8947

Référence électronique

Alain Flajoliet, «O. Külpe, Grundriss des Psychologie», Alter [En ligne], 18 | 2010, mis en ligne le 01 juin 2020, consulté le 24 septembre 2020. URL : http://journals.openedition.org/alter/1808 ; DOI : https:// doi.org/10.4000/alter.1808 


\section{O. KÜLPE, GRUNDRISS DES PSYCHOLOGIE (1893)}

Alain Flajoliet

C'est dans la troisième partie de son ouvrage que Külpe reprend de façon synthétique et thématique tous les fragments où il a été question de l'attention de façon marginale pour produire une conception synthétique de l'attention. Après une définition du phénomène, il examine successivement : 1) les effets, 2) les phénomènes accompagnateurs, 3) les conditions, 4) le caractère volontaire ou non, 5) la liaison au rêve et à l'hypnose, de l'attention. Nous laisserons de côté les points 4 et 5 qui jouent un rôle secondaire dans la doctrine külpienne de l'attention. Rappelons avant de commencer que Külpe se réclame d'abord - si l'on n'envisage que les méthodes d'investigations psychologiques directes - , de la "méthode introspective", avec ses trois réquisits: le sujet qui s'observe doit être attentif, impartial, et être capable de décrire correctement ce qu'il ressent. La «méthode expérimentale » ne peut que compléter l'introspection lorsqu'elle se révèle insuffisante - elle permet de vérifier les descriptions introspectives, de les rendre plus fiables, etc.

On ne trouvera pas chez Külpe de théorie systématique de l'attention elle n'est pas selon lui encore possible- mais plutôt une série d'analyses partielles, de style psychologique descriptif, avec des incursions assez fréquentes dans la psycho-physiologie. Cette modestie le rapproche de Titchener, avec quand même cette différence que son exposé rentre très peu dans les débats critiques avec les principaux psychologues de l'attention, tandis que ces débats occupent une place centrale chez Titchener.

\section{L'attention comme état de conscience}

Külpe commence par une introduction générale dans laquelle il pose la question de la nature de l'attention, qui doit être résolue par la méthode introspective. Il rappelle que d'innombrables théories psychologiques s'opposent sur la définition de cette nature. Certains l'identifient à la 


\section{L'attention}

conscience. D'autres la réduisent à un type particulier de sensations (de tensions, de contractions musculaires, de relâchement), ou à un certain état des centres sensoriels (excitation, inhibition). On peut la considérer comme une émotion exerçant une influence sur le côté moteur de notre activité ; ou, selon une interprétation psychophysique, l'appréhender comme ce qui renforce l'excitation dans les centres sensoriels; ou comme un processus d'inhibition. Le sens commun en fait « une activité interne (innere Tätigkeit) » de la conscience qui s'oriente dans des proportions différentes vers des représentations isolées. Et même lorsqu'un tel changement est involontaire, la croyance reste fort répandue qu'il est au pouvoir de chacun d'être attentif à telle ou telle chose. Il arrive souvent qu'on se réfère à Leibniz qui identifie l'attention avec l' «aperception » (Apperception), c'est-à-dire dit Külpe, avec l'assimilation consciente d'elle-même d'une impression.

Le point décisif touchant à la nature de l'attention, affirme Külpe, c'est qu'elle n'est pas quelque chose qui se situerait «à côté » («neben ») des contenus psychiques et qui s'ajouterait à eux comme un acte spécifique de conscience. La « clarté» («Deutlichkeit oder Klarheit») des contenus est pour lui l'élément fondamental de l'attention (tout comme chez Titchener), mais sa conception a pour originalité d'affirmer fortement que cette qualité appartient uniquement aux contenus et n'est rien d'autre qu'un de leurs attributs possibles. Dès qu'un contenu psychique est clair pour notre conscience, on peut dire que cette dernière y est attentive. Il est donc exact, explique-t-il, d'interpréter comme fonctions de l'attention l'accroissement de la vivacité (Lebhaftigkeit) et de la clarté d'une sensation, ou la concentration de la conscience sur une représentation définie, mais ces changements ne sont pas séparés des contenus psychiques, ils sont en eux. On ne peut donc pas additionner les changements en clarté que subissent les contenus psychiques pour en faire un processus particulier qu'on nommerait « attention » et qui se différencierait des contenus isolés. La discrimination des contenus par l'attention n'est rien de plus qu'une intensification en clarté des attributs des sensations, ou des représentations, ou des relations entre elles, ou une limitation de leur nombre - d'un mot: rien de plus qu'un processus purement quantitatif qui peut être établi dans la conscience par d'autres moyens que l'attention. Mais la clarté et l'attention ne sont-elles pas choses distinctes puisque par exemple un contenu peu intense peut être très clair sous le regard de l'attention? Non, répond Külpe. Si nous faisons abstraction des conditions sensibles de la clarté (comme par exemple le mécanisme d'accommodation des yeux), l'attention se réduit à l'appréhension relativement plus favorable d'une impression, elle-même se réduisant à deux caractères: a) la capacité de l'impression à être discriminée (Unterscheidbarkeit), ce qui renvoie à sa clarté ; b) une tendance particulière des attributs de cette impression à être reproduits (Reproduktionstendenz).

Külpe termine son introduction par quelques considérations générales. La source d'inspiration de toute étude de l'attention, ne peut être selon lui ni la psychologie française, ni la psychologie anglaise, trop marquées par 
l'associationnisme, mais bien la psychologie de Wundt (Physiologische Psychologie 1874, 1903), avec sa distinction entre l'« aperception » (Apperception) et la " perception » (Perception). Selon Wundt, " posséder une représentation » (" eine Vorstellung haben »), n'est pas la même chose que l'éprouver " dans un vivre attentif » ("nicht identisch ist mit einem aufmerksamen Erleben»), les conditions de l'attention ne s'identifient pas aux stimuli internes et externes qui fondent les sensations excitées périphériquement ou centralement. C'est pourquoi il faut distinguer, d'une part, la «perception » qui désigne l'apparition d'un contenu à la conscience à l'occasion de stimuli internes ou externes, et $l^{\prime}$ «apprésentation» qui désigne sa réception dans l'état d'attention. Si pour Külpe, cette distinction est bien fondée, en revanche, la distinction entre attention sensible et intellectuelle lui paraît peu pertinente. Où trouver un critère fiable permettant d'opérer la distinction? Aucun de ceux proposés n'est convaincant. Soit par exemple le critère qui définit l'attention sensible comme se produisant dans les sensations excitées périphériquement, et l'attention intellectuelle comme se produisant dans les sensations excitées centralement. En fait, rien ne prouve que le processus d'excitation soit différent dans les deux cas, sauf à supposer ce qui est justement en question: l'existence de deux formes d'attention. D'une manière analogue, Külpe conteste la valeur de la distinction classique entre attention volontaire et involontaire. Tout au plus accepte-t-il de suivre Wundt lorsque ce dernier distingue aperception (attention) passive et active parce que ce n'est pas une distinction dans le processus d'attention luimême, mais seulement dans ses conditions: l'aperception passive est déterminée de façon non équivoque, et l'aperception active est déterminée de façon équivoque.

Trois tâches essentielles restent à accomplir après la définition de la nature de l'attention. D'abord, décrire les effets de l'attention. Ensuite expliciter les phénomènes secondaires (par exemple, les sensations de tension, de relâchement, etc.) qui accompagnent l'attention. Enfin décrire les conditions d'apparition de l'attention.

\section{Les effets de l'attention}

Constatons d'abord que l'attention induit certaines modifications dans la vie psychique. Külpe revient sur quelques phénomènes importants déjà analysés dans l'ouvrage. D'abord sur les rapports entre sensibilité et attention. Les sensations ont été définies par leurs quatre caractères de qualité, intensité, durée, extension, mais aussi par le fait que la conscience a) les ressent b) les discrimine. L'attention a des effets, d'une part sur la « capacité des sensations à être ressenties" (Empfindlichkeit) - elle accentue cette "sensibilité " et d'autre part sur la «capacité discriminatrice de la sensibilité » (Unterschiedsempfindlichkeit) - ici aussi elle l'accentue. Par ailleurs, l'attention a aussi des effets sur la tendance à la reproduction des sensations 


\section{L'attention}

et sur la fidélité de cette reproduction. Enfin son rôle est négatif sur le sentiment parce qu'elle en affaiblit l'intensité, mais il est positif lorsqu'elle agit sur la formation de la perception d'une fusion de sensations, par exemple sonores.

Peut-on mesurer l'influence de l'attention dans ces différents cas de modifications de la vie psychique? Il n'existe pas encore de méthode rigoureuse pour effectuer cette mesure (voir les Lectures de Titchener). D'abord, il n'existe pas de point fixe dans les variations de l'attention (attention zéro ou attention infinie), ensuite les différences individuelles sont considérables, enfin l'efficacité des moyens employés pour diminuer l'attention portée à des contenus particuliers est trop incertaine. Il existe cependant une estimation subjective du degré de l'attention, obtenue partiellement sur la base des résultats accomplis sous son influence dans les domaines de la perception ou de la pensée, et partiellement sur la base de l'intensité des sensations accompagnatrices de tension. Mais les moyens de mesure objective sont difficiles à trouver. Külpe cite la méthode des stimuli provoquant une distraction : l'attention portée à un contenu sera inversement proportionnelle à la grandeur de la distraction, elle-même mesurée par des modifications en intensité des stimuli qui induisent la distraction. Cependant cette relation entre le stimulus qui détourne l'attention et l'attention elle-même n'est pas aussi simple qu'il n'y paraît. Dans une expérience de discrimination de sons, où le stimulus distrayant était une décharge électrique intense portée au bras, on n'a pas constaté de baisse notable du niveau de discrimination; en revanche parcourir en pensée un morceau de musique pendant un exercice d'évaluation des distances visuelles a réduit considérablement la finesse de la discrimination. Il en ressort qu'une opération intellectuelle perturbe plus l'attention que l'observation d'un stimulus simple. Külpe conclut qu'il n'existe pas (encore) de méthode fiable de mesure des effets de l'attention. On peut seulement décrire par l'introspection certains phénomènes concrets où ces effets sont manifestes.

Passons à l'étude rapide des principaux effets de l'attention. Külpe en relève huit, d'importance plus ou moins grande.

a) L'attention influe sur la "sensibilité » (Empfindlichkeit). Comme cela vient d'être dit, l'attention portée à une sensation la rend plus sensible à la conscience, elle gagne en clarté par rapport au champ des autres sensations. Toutefois les seuils sensoriels sont relativement peu modifiés lorsque l'attention change (lorsque par exemple entrent en scène des stimuli perturbateurs). Külpe ajoute une précision sur les sensations indirectes (ou reproduites) qu'il assimile aux jugements. La sensibilité indirecte est beaucoup plus susceptible d'être modifiée par l'attention que la sensibilité directe. (Cela vaut aussi pour la capacité discriminatrice). Un changement dans les attributs des sensations (qualité, intensité, etc.) ou dans leurs les relations est pour la sensibilité directe confiné dans d'étroites limites, tandis qu'il n'y a quasiment pas de limites aux changements des jugements, c'est-à-dire des reproductions de sensations (sensibilité indirecte). Külpe 
termine par une dernière remarque. L'affirmation que l'attention serait incapable d'intensifier une impression et que le changement observé serait seulement un changement de clarté, est contredite par le fait que la sensation d'un son fort, expérimentée dans l'inattention, peut paraître égale à celle d'un son faible expérimentée attentivement. L'altération du jugement (sensation indirecte) par l'inattention est justement identique à celle produite par une réduction de la valeur intensive (temporelle ou spatiale) des impressions.

b) L'attention influe aussi sur la "capacité discriminatrice de la sensibilité » (Unterschiedsempfindlichkeit), et ce dans deux directions : d'une part elle a des effets sur les différences perçues entre les sensations, et d'autre part elle a des effets sur les jugements portés sur ces sensations. Mais la psychologie est loin encore de savoir bien distinguer entre ces deux effets. Quoi qu'il en soit, il ne faut jamais oublier, comme c'est une tendance trop répandue lorsqu'on étudie les faits de discrimination sensible, que ces derniers sont fortement influencés par l'attention.

c) Attention et reproduction. L'attention a des effets sur la «tendance » des sensations «à être reproduites» (Reproduktionstendenz) et sur la fidélité de cette reproduction. C'est dans cette sphère de la reproduction que l'attention produit ses effets les plus importants, au point que l'on peut se demander si reproduire un vécu sans y faire attention est possible.

d) L'attention influe sur les "sentiments » (Gefühle). L'effet de l'attention sur les sentiments est très spécifique, on l'a vu. L'hypothèse fondamentale de Külpe est celle-ci. Lorsqu'on se concentre attentivement sur le plaisir et la douleur en rendant leurs sensations concomitantes plus vives, ce plaisir et cette douleur disparaissent entièrement si nous parvenons (tout au plus pendant un moment) à porter l'attention sur eux. En ce sens, l'attention entretient un rapport avec les sentiments quasi opposé à celui qu'elle entretient avec les sensations : elle affaiblit la clarté du contenu affectif, elle augmente la clarté du contenu sensoriel.

e) Attention et analyse. L'attention aide considérablement à analyser les groupes de sensations fusionnées (comme un accord sonore, ou une mélodie).

f) Attention, conscience de l'espace et du temps. L'attention permet d'analyser les parties de l'espace, comme les éléments d'une figure géométrique, et les moments d'une série temporelle (mélodie). Elle permet de se concentrer sur ces parties et moments, son étroitesse vient du fait qu'elle ne voit bien que ce qui est limité.

g) Attention, champ, focalisation. Külpe valide les hypothèses de Wundt sur le champ perceptif et le point de fixation de l'attention dans ce champ. $\mathrm{L}^{\prime}$ « aperception » d'un contenu l'extrait du champ sensoriel (Blickfeld) et l'installe au point de fixation (Blickpunkt) de l'attention. Et lorsque l'attention se tourne vers un autre contenu, le contenu initial retombe dans le champ indifférencié et obscur.

h) L'attention et la préséance (Bevorzug) des contenus de conscience. La rapidité avec laquelle un contenu est perçu est proportionnelle à la concentration de l'attente ou de l'attention volontaire qui se portent sur lui. 


\section{L'attention}

Par exemple, lorsque deux stimuli sont perçus simultanément, l'un visuel, l'autre auditif, normalement la sensation visuelle est perçue avec un léger retard. Mais ce retard peut être contrebalancé en focalisant son attention sur la sensation visuelle (voir l'expérience de Geiger). La rapidité de réaction, en outre, est directement fonction du degré d'attention préparatoire.

Külpe conclut son étude des effets de l'attention par quelques remarques plus générales. Il affirme que les effets de l'attention sont fondamentalement de deux sortes, selon qu'ils portent sur les sensations et leurs attributs, ou qu'ils portent sur la reproduction de ces sensations. Dans le premier cas, l'effet essentiel consiste en une intensification, dans le second un accroissement de la tendance à être reproduite et un renforcement de l'associabilité des contenus reproduits.

\section{Phénomènes accompagnant l'attention}

Külpe en analyse quatre.

a) Phénomènes respiratoires et moteurs concomitants. L'accélération du pouls dans la sensation de plaisir décroit quand l'attention se focalise sur cette sensation. Le ralentissement du pouls dans la sensation de déplaisir se renforce quand l'attention se porte sur cette sensation. Autre phénomène intéressant. On retient sa respiration quand on veut se concentrer sur un stimulus, en particulier lorsqu'il est faible ou bref, ou perturbé par d'autres stimuli. Enfin on observe que les mouvements corporels ralentissent dans les phases ou l'attention augmente.

b) Phénomènes adjuvants. Citons parmi eux: l'hyperémie fonctionnelle cérébrale, les sensations à la surface de la peau de pression, de température, de piqûre, parfois de douleur; tous ces phénomènes sont liés à des processus vaso-moteurs.

c) La "vivacité » (Lebhaftigkeit) des contenus sur lesquels se porte l'attention. Ce phénomène touche aussi au rapport entre imagination et attention. Certains observateurs fiables déclarent qu'ils peuvent produire à volonté des sensations visuelles d'une qualité définie et aussi vivantes que celles de la perception externe. Ces qualités visuelles sont donc clairement distinctes des images de la mémoire (elles ont une sorte de présence qui les rapproche des qualités perçues). Dans certains cas extrêmes, elles donnent même lieu à des images consécutives, ce qui signifie que les organes périphériques sont impliqués dans leur formation. Reste que le mécanisme de cette attention est obscur. Les connexions associatives doivent sans doute jouer un rôle. En effet, l'effort pour voir une couleur particulière est confirmé au début par certains jugements - des mots prononcés, entendus ou simplement pensés - qui suffisent à exciter les organes des sens en raison de leur tendance à être reproduits et renforcés par une direction appropriée de l'attention. L'attention volontaire doit récolter un avantage particulier de l'activité de ce mécanisme. Car si la concentration continue de l'attention fait naître des 
sensations actuelles, l'appareil sensoriel physiologique doit être en même temps rendu particulièrement sensible aux impressions externes (on peut comprendre par là que le caractère vivant et concret de l'attention constitue la condition la plus favorable de la perception).

d) L'adaptation des organes des sens. Elle est souvent involontaire comme la contraction de la peau dans les expériences d'attention aux stimuli cutanés. La sensation de tendre l'oreille, qui implique très probablement le tenseur du tympan, accompagne la concentration sur des sons difficiles à discriminer. L'adaptation oculaire est un autre excellent exemple. Non seulement les positions et les mouvements binoculaires dépendent de leur utilité pour la vision attentive, mais le mécanisme de l'accommodation assure les conditions les plus favorables de l'attention volontaire ou involontaire. Külpe ajoute une remarque touchant aux rapports entre l'habitude, qui favorise l'adaptation des sens, et l'attention. L'habitude peut créer une prédisposition sensori-motrice pour l'attention, qui se manifeste par la formation dans les centres inférieurs d'une disposition à la continuation correcte de la sensation ou du mouvement.

Külpe termine par une interrogation : les phénomènes accompagnateurs sont-ils inhibiteurs ou adjuvants? Il laisse la question ouverte. Les phénomènes évoqués en a), b), c), dit-il, sont de nature adjuvante (l'hyperémie fonctionnelle cérébrale favorise l'attention, de même que le fait d'accommoder visuellement, etc.), les phénomènes évoqués en (a) sont plutôt de nature inhibitrice (ralentissement de la respiration, de l'agitation corporelle, etc.)

\section{Les conditions de l'attention}

Ce ne sont pas les conditions de l'état d'attention comme tel qui intéressent Külpe. Ce sont les motifs qui conduisent à l'apparition de contenus particuliers dans l'état attentif. La question est donc celle des circonstances qui attirent l'attention sur ces contenus particuliers. Car l'attention est toujours occupée et en alerte pendant les heures de vigilance de la conscience. Elle est en quelque sorte la cour suprême de la conscience à laquelle peu de processus de la conscience inférieure ont accès. Mais nous distinguons aussi différents degrés d'attention, selon l'intensité de concentration ou la fermeté de sa prise sur les contenus variés. Il faut donc examiner les conditions de ces degrés et de cette fermeté. Külpe analyse successivement les conditions externes et internes. Les premières sont les différents processus moteurs et sensoriels qui donnent à un contenu un avantage sur les autres. Les secondes représentent la valeur affective de l'impression et sa relation aux phénomènes soit présents soit passés de l'histoire de la conscience. Toutes ces conditions valent d'abord pour l'attention involontaire, mais elles influencent aussi l'attention volontaire. 


\section{L'attention}

\section{a) Conditions externes de l'attention, motrices et sensorielles}

Concernant l'aspect moteur, qu'il ne faut pas surestimer, il se présente à la fois sous l'aspect de mouvements expressifs et sous l'aspect de processus à proprement parler moteurs permettant de former et de maintenir l'attention. L'équipement moteur possède en effet des prédispositions à recevoir tel ou tel type de stimulus. Toutes choses égales par ailleurs, l'attention est plus susceptible d'être attirée par un objet qui (occasionnellement ou intentionnellement) a été provoqué par les conditions motrices de la vision claire (fixation et accommodation). La position du corps joue aussi un rôle. Il est douteux que ces facteurs moteurs constituent une condition spéciale de l'attention au-delà des effets sensibles qu'ils provoquent. Mais ils sont indirectement favorables à l'aperception des contenus particuliers en tant qu'ils déterminent les attributs des contenus eux-mêmes.

Concernant les conditions sensorielles de l'attention, elles incluent tous ces attributs des sensations excitées périphériquement ou centralement qui leur donnent une influence sur l'aperception, indépendamment des relations qu'elles ont avec les autres contenus simultanément présents dans la conscience. Le premier attribut est négatif, c'est l'absence d'autres impressions. Plus un son (ou une perception visuelle, etc.) est isolé, plus il a la force d'attirer l'attention. Cette observation se relie aux faits de "concentration " (Konzentration) de l'attention, et à $l^{\prime}$ «étroitesse» (Enge) de la conscience. Elle explique en outre probablement, dit Külpe, les "effets de contraste » (Kontrastwirkungen $^{1}$ ). La cause de cet effet est sans doute en effet qu'une impression sensible focalise l'attention plus aisément si elle est isolée d'un environnement, que si elle est prise dans un environnement. Or, justement, un effet de contraste consiste dans une facilité particulière à porter l'attention sur un objet en le détachant de son environnement, bref dans une facilité à isoler une impression.

Le second attribut (cette fois-ci positif) c'est l'intensité de l'impression. Des impressions relativement intenses attirent l'attention dans tous les domaines sensoriels comme si le stimulus intense avait le pouvoir de pénétrer dans le " cercle magique » que la conscience supérieure (das Oberbewusstsein) a élevé autour d'elle-même. En considérant l'apparition précoce d'une relation entre les impressions sensorielles et les mouvements réflexes au cours du développement individuel, on ne peut douter du caractère biogénétique de ce second attribut.

1. Külpe a étudié les effets de contraste un peu plus haut dans son livre (§ 68.7). Rappelons par exemple cette loi du "contraste de la luminosité » (Helligkeitscontrast). Quand deux luminosités de qualités différentes sont vues simultanément ou successivement, la différence entre elles est accrue en apparence. Si on a devant soi un petit carré blanc sur une surface blanche d'une qualité identique, un carré analogue sur une surface grise, et un troisième carré de même sorte sur un fond noir, le premier carré apparaît plus sombre (son intensité n'est pas avivée par l'absence de contraste), le dernier carré apparaît plus clair (son intensité est avivée par contraste avec le noir), la clarté du second papier étant médiane. 


\section{b) Conditions internes de l'attention}

Elles sont essentiellement de deux sortes : affectives et psychophysiques.

Conditions affectives. L'attention se porte préférentiellement sur les impressions ayant un "effet sur l'affectivité" (Gefühlswirkung) et qui provoquent l'intérêt (Interesse) de la conscience. Ce qui a conduit parfois à identifier attention et intérêt, donc à en faire simplement un sentiment. Position inexacte pour Külpe, parce qu'une frontière stricte sépare l'attention et le sentiment. Voici son argument, qui s'appuie sur les sentiments fondamentaux de l'agréable et du désagréable. En règle générale, on constate qu'une impression agréable attire notre attention autant qu'une impression désagréable. De cette constatation banale, on peut tirer la conclusion que le vécu attentionnel et le vécu affectif sont des choses très différentes. Imaginons que j'éprouve d'abord un sentiment agréable avec un certain degré d'attention, puis un sentiment désagréable avec exactement le même degré d'attention. L'attention n'a pas varié, le sentiment a totalement changé, preuve que, au moins dans ce cas, ils ne sont pas identiques. Külpe généralise : ils ne sont jamais identiques, les théories identifiant attention et intérêt sont fausses.

\section{Le rapport d'une impression à la "disposition psychophysique »} (psychophysische Disposition)

Külpe dénombre trois sortes de rapports, qui représentent toujours des conditions primordiales de l'attention. D'abord, les relations associatives aux représentations déjà présentes dans la conscience. Ensuite, les relations aux fondements de la reproduction, c'est-à-dire à ces dispositions préalables permettant l'entrée de l'impression dans le champ de l'attention. Enfin, la vacuité relative de la conscience.

Commençons par le premier type de rapport. Plus une impression possède une « tendance à être reproduite » (Reproduktionstendenz), plus aisément elle attire l'attention. Tout apprentissage efficace doit s'inspirer de cette loi. Pour être assimilée, une matière doit être connectée avec l'expérience passée, soit par sa similarité avec ce qui est déjà su, soit par quelque autre motif de reproduction. Si l'impression a été vécue, puis reproduite une fois, deux fois, etc. elle acquiert une capacité d'activation qui favorise sa captation par l'attention par rapport à des représentations nouvelles. La sédimentation des expériences où on l'a expérimentée et ré-expérimentée en fait un point de fixation favorable. L'attention volontaire peut utiliser ces relations associatives pour l'aperception d'impressions particulières ou de représentations. Ici aussi des impressions semblables ou identiques à des représentations déjà présentes dans la conscience sont particulièrement susceptibles d'attirer l'attention. Portées en effet par une tendance à être reproduites qui leur donne une capacité de ressurgir à tout instant, elles sont particulièrement aptes à repousser les autres contenus hors du point de fixation de l'attention. 


\section{L'attention}

Passons au second type de rapport. Des contenus semblables ou identiques ont été déjà expérimentés auparavant et ont créé des « dispositions fonctionnelles » (funktionnelle Dispositionen) qui facilitent leur fixation par la conscience attentive. Les représentations qui surgissent librement dans la conscience sont de ce type. Elles suscitent l'attention en raison de la vivacité (Lebhaftigkeit) et de la persistance (Andauer) de leurs matériaux de reproduction, indépendamment de leurs attributs ou de leurs relations. Le pouvoir des sensations excitées centralement d'attirer l'attention varie selon le niveau de préparation auquel il a été a été relevé par la disposition psychophysique. C'est la raison pour laquelle nous pouvons aisément entendre à part un harmonique dans un accord si nous l'avons auparavant entendu seul; ou que l'histologiste trouve dans sa préparation une foule de détails intéressants qui échappent à l'œil inexercé. Tout ceci se transpose à l'attention volontaire, comme lorsque nous stimulons aussi intensément que possible les matériaux de la reproduction d'une impression attendue. Le temps de préparation est à peu près constant, entre deux secondes et deux secondes et demie.

Terminons par le troisième rapport. L'attention est conditionnée par la vacuité relative de la conscience. Cela se comprend aisément, on a vu que parmi les conditions externes d'émergence d'une impression à l'attention, il fallait compter l'absence d'impressions rivales risquant de capter l'attention à leur profit. Autrement dit, un contenu tombe d'autant plus aisément sous le regard de l'attention que cette dernière n'est pas déjà occupée par d'autres contenus.

Külpe termine son exposé par quelques lignes touchant aux «fluctuations de l'attention » (Schwankungen der Aufmerksamkeit). Il en donne une explication psycho-physiologique plutôt qu'une description purement introspective. Tout stimulus, dit-il, qui persiste au-delà d'une certaine durée finit par perdre de sa force. Des travaux ont été menés pour quantifier les lois temporelles auxquelles obéit l'attention, en observant par exemple les variations du seuil différentiel au cours du temps - la décroissance de ce seuil aboutit à un point zéro (plus aucune différence n'est perçue entre les deux sensations). Mais en prolongeant encore l'expérience, il y a un retour de la conscience de seuil. Et de nouveau une décroissance de cette conscience. L'origine de ces fluctuations se situe pour Külpe dans la sensibilité centrale et non périphérique.

De ces dernières remarques, mais aussi de l'ensemble de l'ouvrage, il ressort que la démarche de Külpe est loin d'être purement introspective, en dépit des intentions affichées. Lorsqu'il conclut son étude de l'attention pour esquisser ce que pourrait être une théorie correcte de cette fonction psychique, ses préférences vont nettement à une théorie solidement arrimée aux connaissances psychophysiques. Si par exemple selon lui les théories de Ribot et de Herbart sont contestables, c'est parce que cet ancrage dans la psychophysiologie leur fait défaut. En revanche, affirme-t-il, les travaux de G.E. Müller, même si leurs résultats méritent discussion, reposent sur une méthode d'investigation solide fondée sur l'analyse psychophysiologique 


\section{Recensions}

(de fait, Müller définit l'attention comme un renforcement de sensations ou de représentations excitées périphériquement). Pour Külpe la méthode utilisée pour définir ici l'attention est correcte, mais le contenu de la définition reste discutable parce que Müller passe à côté des phénomènes inhibiteurs qui jouent un rôle absolument fondamental dans l'attention. 